This is a postprint version of the following published document:

Cassatella-Contra, G. A.; Mañas, M.; Tempesta, P. (2014).

"Singularity confinement for matrix discrete Painlevé

Equations". Nonlinearity, 27, pp. 2321-2335.

DOI: $10.1088 / 0951-7715 / 27 / 9 / 2321$

(C) IOP Publishing 2014 


\title{
Singularity confinement for matrix discrete Painlev'e equations
}

\author{
Giovanni A Cassatella-Contra ${ }^{1}$, Manuel Mañas ${ }^{1}$ and \\ Piergiulio Tempesta ${ }^{1,2}$ \\ ${ }^{1}$ Departamento de Física Teórica II (Métodos Matemáticos de la Física), Facultad de Físicas, \\ Universidad Complutense de Madrid, 28040 Madrid, Spain \\ ${ }^{2}$ Instituto de Ciencias Matemáticas, C/ Nicolás Cabrera, No 13-15, 28049 Madrid, Spain
}

E-mail: p.tempesta@fis.ucm.es, gaccontra@fis.ucm.es and manuel.manas@ucm.es

\begin{abstract}
We study the analytic properties of a matrix discrete system introduced by Cassatella and Ma nas (2012 Stud. Appl. Math. 128 252-74). The singularity confinement for this system is shown to hold generically, i.e. in the whole space of parameters except possibly for algebraic subvarieties. This paves the way to a generalization of Painlev'e analysis to discrete matrix models.
\end{abstract}

Keywords: singularity confinement, discrete integrable systems, noncommu-tative discrete Painlev'e I equation, Schur complements

\section{Introduction}

Since the discovery of the Painlevé property for ordinary differential equations at the end of the 19th century [21], the notion of integrability has been related to the local analysis of movable isolated singularities of solutions of dynamical systems [8]. This approach to integrability has opened an alternative perspective compared with the standard algebraic approach $\grave{a} l a$ Liouville, based on the existence of a suitable number of functionally independent integrals of motion. Both points of view have been extended to the study of evolution equations on a discrete background.

Integrable discrete systems, for several aspects more fundamental objects than the continuous ones, are ubiquitous in both pure and applied mathematics, and in theoretical physics as well. They possess rich algebro-geometric properties $[3,5,9,18,25]$ and are relevant, for instance, in the regularization of quantum field theories in a lattice and in discrete quantum gravity $[10,16]$. 
In particular, the problem of integrability preserving discretizations of partial differential equations has become a very active research area [23], and has been widely investigated with both geometrical and algebraic methods [5, 6, 20,24].

The approach known as singularity confinement, introduced in [13], is the equivalent for discrete systems of the singularity analysis for continuous dynamical systems. It essentially relies on the observation that for integrable discrete models, if a singularity appears in some specific point of the lattice of the independent variable, then it would disappear after making the system evolve via a finite number of iterations. Alternative, related approaches are based on the notion of algebraic entropy [4,17] or on Nevalinna theory [1,22]. A large class of difference equations coming from unitary integrals and combinatorics possess the confinement property [2]. However, observe that singularity confinement, in spite of being extremely useful in isolating integrability, might not be a sufficient condition for integrability, as was observed by Hietarinta and Viallet [15].

The purpose of this paper is to start a theoretical study of the singularity confinement property for matrix integrable systems. Indeed, we hypothesize that the singularity analysis has the same relevance for matrix systems that it possesses for both discrete and continuous scalar models.

Apart from its intrinsic mathematical interest, the study of matrix discrete dynamical systems can also be related, from an applicative point of view, to the theory of complex networks [19]. Indeed, given a random graph with $N$ vertices, one associates with it the adjacency matrix, which is a $N \times N$ matrix, whose entries $a_{i j}$ represent the number of links associated with the nodes $i$ and $j(i, j=1, \ldots, N)$. The discrete time evolution of the topology of the network would provide a difference equation for the adjacency matrix, defining a discrete matrix model.

Hereafter, we shall focus on the singularity confinement of the following discrete matrix equation

$$
\beta_{n+1}=n \beta_{n}^{-1}-\beta_{n-1}-\beta_{n}-\alpha, \quad n=1,2, \ldots
$$

where $\beta_{n} \in \mathbb{C}^{N \times N}$ is a $N \times N$ complex matrix.

Equation (1) can be considered a kind of non-Abelian matrix version of the discrete Painlevé equation (dPI). It was introduced in [7], and soon after studied in [14], and describes the recursion relation for the matrix coefficients of a class of Freud matrix orthogonal polynomials with a quartic potential [11] in the context of the associated Riemann-Hilbert problem. In that paper we also proved the singularity confinement in a simple situation, when the initial data are triangular matrices up to similarity transformations. The aim of this paper is to extend this result to the general case. This extension relies heavily on the use of Schur complements, which appear often in the analysis of non-Abelian systems, see [12]. It should also be remarked that this proof required deeper understanding and study than in the triangularizable situation. The difficulty mainly resides in the analysis of the genericness of the result given in theorem 2 .

\subsection{Preliminary discussion}

Let us present here the simplest case of singularity analysis for the matrix model (1), which parallels the results for the standard discrete Painlevé I equation. We assume that $\beta_{m-1}$ do not depend on $\epsilon$ and that

$$
\beta_{m}=\beta_{m, 1} \epsilon+\beta_{m, 2} \epsilon^{2}+O\left(\epsilon^{3}\right), \quad \epsilon \rightarrow 0,
$$

with det $\beta_{m, 1} \neq 0$. Observe that we are assuming the leading term for $\beta_{m}$ is proportional to $\epsilon$, we say that we have a 'zero'. Note also that the leading term coefficient is required, in this example, to be invertible. This is the only possibility in the scalar case $N=1$, but as we will 
discuss later the non-Abelian scenario $N \geqslant 2$ implies a richer situation. Thus, as this approach will hold hereon, we assume that at some integer $m$ of the lattice a zero appears, while for the previous one, $m-1$, neither a zero nor singularity shows up.

If we introduce condition (2) into (1), we have that

$$
\beta_{m+1}=m \beta_{m, 1}^{-1} \epsilon^{-1}+\beta_{m+1,0}+\beta_{m+1,1} \epsilon+\beta_{m+1,2} \epsilon^{2}+O\left(\epsilon^{3}\right),
$$

where

$\beta_{m+1,0}=-m \beta_{m, 1}^{-1} \beta_{m, 2} \beta_{m, 1}^{-1}-\beta_{m-1}-\alpha$,

$\beta_{m+1,1}=m \beta_{m, 1}^{-1}\left(\beta_{m, 2} \beta_{m, 1}^{-1} \beta_{m, 2}-\beta_{m, 3}\right) \beta_{m, 1}^{-1}-\beta_{m, 1}$,

$\beta_{m+1,2}=m\left(\beta_{m, 2} \beta_{m, 1}^{-1}\left(\beta_{m, 3}-\beta_{m, 2} \beta_{m, 1}^{-1} \beta_{m, 2}\right)+\beta_{m, 3} \beta_{m, 1}^{-1} \beta_{m, 2}-\beta_{m, 4}\right) \beta_{m, 1}^{-2}-\beta_{m, 2}$.

We observe that a leading term in $\epsilon^{-1}$ appeared in the asymptotic expansion. This 'pole singularity' will survive still for another step in the sequence

$$
\beta_{m+2}=-m \beta_{m, 1}^{-1} \epsilon^{-1}+\beta_{m+2,0}+\beta_{m+2,1} \epsilon+\beta_{m+2,2} \epsilon^{2}+O\left(\epsilon^{3}\right)
$$

where

$$
\begin{aligned}
\beta_{m+2,0}= & m \beta_{m, 1}^{-1} \beta_{m, 2} \beta_{m, 1}^{-1}+\beta_{m-1}, \\
\beta_{m+2,1}= & \frac{(m+1)}{m} \beta_{m, 1}-m \beta_{m, 1}^{-1} \beta_{m, 2} \beta_{m, 1}^{-1} \beta_{m, 2} \beta_{m, 1}^{-1}+m \beta_{m, 1}^{-1} \beta_{m, 3} \beta_{m, 1}^{-1}, \\
\beta_{m+2,2}= & \frac{(m+1)}{m} \beta_{m, 2}+\frac{(m+1)}{m^{2}} \beta_{m, 1}\left(\beta_{m-1}+\alpha\right) \beta_{m, 1} \\
& +m \beta_{m, 2} \beta_{m, 1}^{-1}\left(\beta_{m, 2} \beta_{m, 1}^{-1} \beta_{m, 2} \beta_{m, 1}^{-2}-\beta_{m, 3} \beta_{m, 1}^{-2}\right)-m \beta_{m, 3} \beta_{m, 1}^{-1} \beta_{m, 2} \beta_{m, 1}^{-2}+m \beta_{m, 4} \beta_{m, 1}^{-2} .
\end{aligned}
$$

We easily check that in the third step the leading term is proportional to $\epsilon$, this 'zero' appears again

$$
\beta_{m+3}=\frac{-(m+3)}{m} \beta_{m, 1} \epsilon+\beta_{m+3,2} \epsilon^{2}+O\left(\epsilon^{3}\right)
$$

where

$$
\beta_{m+3,2}:=-\frac{(m+3)}{m} \beta_{m, 2}-\frac{(2 m+3)}{m^{2}} \beta_{m, 1} \beta_{m-1} \beta_{m, 1}-\frac{(m+1)}{m^{2}} \beta_{m, 1} \alpha \beta_{m, 1} .
$$

Finally, if we substitute (4) and (5) into (1) we obtain no singularity at all:

$$
\beta_{m+4}=\frac{m}{(m+3)} \beta_{m-1}-\frac{2}{(m+3)} \alpha+O(\epsilon)
$$

Observe that $\beta_{m+3}=O(\epsilon), \beta_{m+4}=O(1)$ and det $\beta_{m+4}=O(1)$ for $\epsilon \rightarrow 0$. Thus, unless

$$
\operatorname{det}\left(m \beta_{m-1}-2 \alpha\right)=0,
$$

we obtain singularities in the step just after the appearance of a zero in $\beta_{m}$, with the poles appearing in the sites $m+1, m+2$. Then we have a zero for $m+3$ while we recover the standard behaviour for $m+4$. A crucial point is that this singularity confinement holds whenever (6) is not satisfied. This observation motivates the definitions proposed in the following discussion.

Definition 1. Whenever the singularity confinement property is satisfied in the whole space $\mathcal{S}$ of parameters except possibly for a set of algebraic subvarieties $\mathcal{W}_{i} \in \mathcal{S}, i=1,,, j \in \mathbb{N}$, we shall say that the property is satisfied generically.

In this case we will speak about the genericness of the singularity confinement. 
Definition 2. We shall define the confinement time as the minimum number $l \in \mathbb{N}$ of iterations or steps in the lattice, after the appearance of a zero, necessary to recover the form without poles or zeros.

Thus, in the above case we have generically a singularity confinement with a confinement time $l=4$.

A simple but fundamental observation for the sequel of the paper is the following one.

Lemma 1. The matrix system (1) is invariant under similarity transformations.

Proof. Observe that

$$
M \beta_{n+1} M^{-1}=n M \beta_{n}^{-1} M^{-1}-M \beta_{n-1} M^{-1}-M \beta_{n} M^{-1}-M \alpha M^{-1} .
$$

Therefore, we obtain

$$
\phi_{n+1}=n \phi_{n}^{-1}-\phi_{n-1}-\phi_{n}-\delta
$$

where $\phi_{n}:=M \beta_{n} M^{-1}$ and $\delta:=M \alpha M^{-1}$.

\subsection{Main result}

The ideas developed within the previous example will be used in the subsequent considerations to study the confinement of the singularities of the matrix dPI model (1). In this noncommutative scenario we must be careful when we talk about zeroes and singularities associated with asymptotic expansions. For the example discussed above it was just as in the Abelian case with $N=1$ as we assumed that the leading term coefficients of the zero was an invertible matrix. In general this is just not the case and we need to consider the rank, $\operatorname{rank}\left(\beta_{m, 0}\right)$, of the matrix coefficient of the leading term of $\beta_{m}$.

As before let us suppose that for some integer $m$ of the lattice a zero appears, while for $(m-1)$ neither a zero nor singularity shows up. But now we must carefully explain what we mean by a zero. We shall assume that $\beta_{m-1}$ do not depend on $\epsilon$ and that

$$
\beta_{m}=\beta_{m, 0}+\beta_{m, 1} \epsilon+O\left(\epsilon^{2}\right), \quad \operatorname{det} \beta_{m}=O\left(\epsilon^{r}\right), \quad \epsilon \rightarrow 0,
$$

where $\beta_{m, i} \in \mathbb{C}^{N \times N}$ and $r \in\{1, \ldots, N\}$. Consequently, we can distinguish two cases.

- $r=N$. This is the maximal rank case discussed above; for it we have that

$$
\beta_{m, 0}=0, \quad \operatorname{det} \beta_{m, 1} \neq 0 .
$$

As we have already seen it presents singularity confinement generically.

- $r \leqslant N-1$. For the non-maximal rank case we instead have

$$
\begin{aligned}
& \operatorname{rank}\left(\beta_{m, 0}\right)=N-r, \\
& \operatorname{det} \beta_{m}=O\left(\epsilon^{r}\right), \quad \epsilon \rightarrow 0 .
\end{aligned}
$$

As will be proven later, using the invariance under a similarity transformation, one can assume that the matrices $\beta$ will have the form expressed by equation (13). So said, we can state the main result of the paper as follows.

Theorem 1. If $\beta_{m-1}$ do not depend on $\epsilon$ and $\beta_{m}$ is of the form (7), and the following conditions for $\epsilon \rightarrow 0$ are satisfied

$$
\begin{aligned}
& \operatorname{det} \beta_{m+1}=O\left(\epsilon^{-r}\right), \\
& \operatorname{det} \beta_{m+2}=O\left(\epsilon^{-r}\right), \\
& \operatorname{det} \beta_{m+3}=O\left(\epsilon^{r}\right), \\
& \operatorname{det} \beta_{m+4}=O(1),
\end{aligned}
$$

then, there is singularity confinement for the dPI model (1) with confinement time $l=4$. 
It is important to remark that conditions (9)-(12) can be proven to hold generically, that is the content of theorem (2). Therefore, we can state that our system generically has the singularity confinement property.

\section{2. $N \times N$ matrix asymptotic expansions and singularity confinement}

In this section we will consider the set of matrix asymptotic expansions

$$
\mathcal{A}=\mathbb{C}^{N \times N}((\epsilon)):=\left\{M_{0}+M_{1} \epsilon+O\left(\epsilon^{2}\right), \epsilon \rightarrow 0, M_{i} \in \mathbb{C}^{N \times N}\right\} .
$$

This set is a ring with identity, given by the matrix $\mathbb{I}_{N}$. For each possible rank $r \in\{1, \ldots, N-1\}$ we will use the block notation

$$
M:=\left(\begin{array}{ll}
A & B \\
C & D
\end{array}\right), \quad A \in \mathbb{C}^{r \times r}, B \in \mathbb{C}^{r \times(N-r)}, C \in \mathbb{C}^{(N-r) \times r}, D \in \mathbb{C}^{(N-r) \times(N-r)} .
$$

We also introduce two subalgebras of the algebra $\mathbb{C}^{N \times N}$

$$
\begin{aligned}
& \mathfrak{K}:=\left\{K=\left(\begin{array}{cc}
0 & 0 \\
K_{21} & K_{22}
\end{array}\right), K_{21} \in \mathbb{C}^{(N-r) \times r}, K_{22} \in \mathbb{C}^{(N-r) \times(N-r)}\right\}, \\
& \mathfrak{L}:=\left\{L=\left(\begin{array}{ll}
L_{11} & 0 \\
L_{21} & 0
\end{array}\right), L_{11} \in \mathbb{C}^{r \times r}, L_{21} \in \mathbb{C}^{(N-r) \times r}\right\},
\end{aligned}
$$

and the related subsets of matrix asymptotic expansions

$$
\mathcal{A}_{\mathfrak{K}}:=\left\{K \in \mathcal{A},\left.K\right|_{\epsilon=0} \in \mathfrak{K}\right\}, \quad \mathcal{A}_{\mathfrak{L}}:=\left\{L \in \mathcal{A},\left.L\right|_{\epsilon=0} \in \mathfrak{L}\right\},
$$

which satisfy several important properties.

Proposition 1. The following statements hold.

(1) Both $\mathcal{A}_{\mathfrak{K}}$ and $\mathcal{A}_{\mathfrak{L}}$ are subrings without identity of the ring $\mathcal{A}$.

(2) For $K \in \mathcal{A}_{\mathfrak{K}}$ such that $\operatorname{det} K=O\left(\epsilon^{r}\right), \epsilon \rightarrow 0$, then $K^{-1} \in \epsilon^{-1} \mathcal{A}_{\mathfrak{L}}$, and reciprocally if $L \in \epsilon^{-1} \mathcal{A}_{\mathfrak{L}}$ with $\operatorname{det} L=O\left(\epsilon^{-r}\right), \epsilon \rightarrow 0$, then $L^{-1} \in \mathcal{A}_{\mathfrak{K}}$.

(3) If $K \in \mathcal{A}_{\mathfrak{K}}$, that is $K=\left(\begin{array}{cc}0 & 0 \\ C_{0} & D_{0}\end{array}\right)+\left(\begin{array}{ll}A_{1} & B_{1} \\ C_{1} & D_{1}\end{array}\right) \epsilon+O\left(\epsilon^{2}\right)$ then

$$
\operatorname{det} K=\epsilon^{r} \operatorname{det}\left(\begin{array}{ll}
A_{1} & B_{1} \\
C_{0} & D_{0}
\end{array}\right)+O\left(\epsilon^{r+1}\right), \quad \epsilon \rightarrow 0 .
$$

(4) If $L \in \epsilon^{-1} \mathcal{A}_{\mathfrak{L}}$, that is $L=\left(\begin{array}{ll}A_{0} & 0 \\ C_{0} & 0\end{array}\right) \epsilon^{-1}+\left(\begin{array}{ll}A_{1} & B_{1} \\ C_{1} & D_{1}\end{array}\right)+O(\epsilon)$ then

$$
\operatorname{det} L=\epsilon^{-r} \operatorname{det}\left(\begin{array}{ll}
A_{0} & B_{1} \\
C_{0} & D_{1}
\end{array}\right)+O\left(\epsilon^{-r+1}\right), \quad \epsilon \rightarrow 0 .
$$

(5) The subrings $\mathcal{A}_{\mathfrak{K}}$ and $\mathcal{A}_{\mathfrak{L}}$ are right and left ideals of $\mathcal{A}$, respectively, i.e. $\mathcal{A}_{\mathfrak{K}} \cdot \mathcal{A} \subset \mathcal{A}_{\mathfrak{K}}$ and $\mathcal{A} \cdot \mathcal{A}_{\mathfrak{L}} \subset \mathcal{A}_{\mathfrak{L}}$.

(6) The following inclusion holds: $\epsilon^{-1} \mathcal{A}_{\mathfrak{L}} \cdot \mathcal{A}_{\mathfrak{K}} \subset \mathcal{A}$.

The proof of the previous statements is direct and left to the reader.

To study the singularity confinement of the matrix equation (1) when $\beta_{n}$ satisfies conditions (8), we shall use expressions (7), having applied a similarity transformation to $\beta$ such that 
$\beta_{m, 0} \in \mathfrak{K}, \beta_{m} \in \mathcal{A}_{\mathfrak{K}}$. In other words

$$
\beta_{m, 0}=\left(\begin{array}{ccccccc}
0 & 0 & \cdots & 0 & 0 & \cdots & 0 \\
0 & 0 & \cdots & 0 & 0 & \cdots & 0 \\
\vdots & \vdots & & \vdots & \vdots & & \vdots \\
\beta_{m, 0 ; r+1,1} & \beta_{m, 0 ; r+1,2} & \cdots & \beta_{m, 0 ; r+1, r+1} & \beta_{m, 0 ; r+1, r+2} & \cdots & \beta_{m, 0 ; r+1, N} \\
\beta_{m, 0 ; r+2,1} & \beta_{m, 0 ; r+2,2} & \cdots & \beta_{m, 0 ; r+2, r+1} & \beta_{m, 0 ; r+2, r+2} & \cdots & \beta_{m, 0 ; r+2, N} \\
\vdots & \vdots & & \vdots & \vdots & & \vdots \\
\beta_{m, 0 ; N, 1} & \beta_{m, 0 ; N, 2} & \cdots & \beta_{m, 0 ; N, r+1} & \beta_{m, 0 ; N, r+2} & \cdots & \beta_{m, 0 ; N, N}
\end{array}\right) \text {, }
$$

where $m \geqslant 2$, and all the entries that are above the $r+1$-th row of $\beta_{m}$ are zero. Notice that $\beta_{m-1}$ and $\beta_{m}$ belong to the rings $\mathcal{A}$ and $\mathcal{A}_{\mathfrak{K}}$, respectively.

\subsection{Proof of the theorem 1}

Proof. As $\beta_{m, 0} \in \mathfrak{K}$, i.e. $\beta_{m} \in \mathcal{A}_{\mathfrak{K}}$, and by hypothesis $\operatorname{det} \beta_{m}=O\left(\epsilon^{r}\right), \epsilon \rightarrow 0$, proposition 1 implies

$$
\beta_{m}^{-1}=\left(\beta_{m}^{-1}\right)_{-1} \epsilon^{-1}+\left(\beta_{m}^{-1}\right)_{0}+O(\epsilon), \quad \epsilon \rightarrow 0, \quad\left(\beta_{m}^{-1}\right)_{-1} \in \mathfrak{L} .
$$

If we replace equations (7) and (13) into equation (1) we deduce

$$
\beta_{m+1}=m \beta_{m}^{-1}+O(1), \quad \epsilon \rightarrow 0 .
$$

Using the relations (14), (7) and (13), this expression is reduced to

$$
\beta_{m+1}=m\left(\beta_{m}^{-1}\right)_{-1} \epsilon^{-1}+O(1), \quad \epsilon \rightarrow 0 .
$$

Since $\left(\beta_{m}^{-1}\right)_{-1} \in \mathfrak{L}$, from (15) we conclude that $\beta_{m+1} \in \epsilon^{-1} \mathcal{A}_{\mathfrak{L}}$, showing a simple pole singularity. Due to the fact that by hypothesis equation (9) holds, proposition 1 implies

$$
\beta_{m+1}^{-1} \in \mathcal{A}_{\mathfrak{K}} \text {. }
$$

Then we deduce

$$
\beta_{m+2}=-m\left(\beta_{m}^{-1}\right)_{-1} \epsilon^{-1}+O(1), \quad \epsilon \rightarrow 0, \quad \beta_{m+2} \in \mathcal{A}_{\mathfrak{L}} .
$$

As before, using condition (10), proposition 1 gives

$$
\beta_{m+2}^{-1} \in \mathcal{A}_{\mathfrak{K}} .
$$

Now,

$$
\beta_{m+3}=\beta_{m}-(m+1) \beta_{m+1}^{-1}+(m+2) \beta_{m+2}^{-1},
$$

where in the rhs we have used twice equation (1) to write $\beta_{m+2}$ as a function of $\beta_{m+1}$ and $\beta_{m}$. As we have proven that $\beta_{m}, \beta_{m+1}^{-1}, \beta_{m+2}^{-1} \in \mathcal{A}_{\mathfrak{K}}$, we deduce that

$$
\beta_{m+3} \in \mathcal{A}_{\mathfrak{K}} .
$$

As a consequence of equation (11) and proposition 1, we obtain

$$
\beta_{m+3}^{-1} \in \epsilon^{-1} \mathcal{A}_{\mathfrak{L}} .
$$

Our matrix discrete Painlevé equation (1) gives

$$
\beta_{m+4}=(m+3) \beta_{m+3}^{-1}-\beta_{m+2}-\beta_{m+3}-\alpha,
$$


which implies

$\beta_{m+4}=\beta_{m+3}^{-1} A+O(1), \quad \epsilon \rightarrow 0, \quad A:=(m+3) \mathbb{I}_{N}-\beta_{m+3} \beta_{m+2}$,

where we have taken into account that $\beta_{m+3}$ and $\alpha$ are $O(1)$. We study the matrix $A$, by applying equation (1) once. We obtain

$$
\begin{aligned}
A & =\mathbb{I}_{N}+\left[(m+1) \beta_{m+1}^{-1}-\beta_{m}\right] \beta_{m+2} \\
& =\left[(m+1) \beta_{m+1}^{-1}-\beta_{m}\right]\left[(m+1) \beta_{m+1}^{-1}-\beta_{m}-\alpha\right]-m \mathbb{I}_{N}+\beta_{m} \beta_{m+1} \\
& =\left[(m+1) \beta_{m+1}^{-1}-\beta_{m}\right]\left[(m+1) \beta_{m+1}^{-1}-\beta_{m}-\alpha\right]-\beta_{m}\left(\beta_{m}+\beta_{m-1}+\alpha\right) .
\end{aligned}
$$

Now, recalling that $\beta_{m-1}=O(1), \beta_{m}, \beta_{m+1}^{-1} \in \mathcal{A}_{\mathfrak{K}}$, and by virtue of proposition 1 we conclude that

$$
A \in \mathcal{A}_{\mathfrak{K}} .
$$

Finally, from equations (18), (19) and (21) we deduce that

$$
\beta_{m+4} \in \mathcal{A} \text {. }
$$

By taking into account that $\operatorname{det} \beta_{m+4}=O(1)$, we have proven that the singularity has disappeared. Thus, the singularity confinement is ensured with a confinement time $l=4$.

In order to show the genericness of conditions (9)-(12) we use the block notation

$$
\beta_{m-1}=\left(\begin{array}{ll}
A_{m-1} & B_{m-1} \\
C_{m-1} & D_{m-1}
\end{array}\right), \quad \alpha=\left(\begin{array}{ll}
\alpha_{11} & \alpha_{12} \\
\alpha_{21} & \alpha_{22}
\end{array}\right) .
$$

and consider the expansion

$$
\beta_{m}=\left(\begin{array}{cc}
0 & 0 \\
C_{m, 0} & D_{m, 0}
\end{array}\right)+\sum_{i=1}^{\infty}\left(\begin{array}{cc}
A_{m, i} & B_{m, i} \\
C_{m, i} & D_{m, i}
\end{array}\right) \epsilon^{i} .
$$

Definition 3. We introduce

$$
\begin{aligned}
& Z_{1}:=D_{m+1,0}+D_{m, 0}^{-1} C_{m, 0} B_{m+1,0}, \\
& Z_{2}:=D_{m+2,0}+D_{m, 0}^{-1} C_{m, 0} B_{m+2,0}, \\
& Z_{3}:=D_{m+3,0} .
\end{aligned}
$$

The genericness of the singularity confinement can be stated as follows.

Theorem 2. (1) If det $D_{m, 0} \neq 0$, for $\epsilon \rightarrow 0$ we have

$$
\operatorname{det} \beta_{m+1}=O\left(\epsilon^{-r}\right) \Leftrightarrow \operatorname{det}\left(Z_{1}\right) \neq 0 .
$$

(2) If $\operatorname{det} D_{m, 0} \neq 0, \operatorname{det} Z_{1} \neq 0$, we have that for $\epsilon \rightarrow 0$

$$
\operatorname{det} \beta_{m+2}=O\left(\epsilon^{-r}\right) \Leftrightarrow \operatorname{det}\left(Z_{2}\right) \neq 0 .
$$

(3) If $\operatorname{det} D_{m, 0} \neq 0, \operatorname{det} Z_{1} \neq 0$ and $\operatorname{det} Z_{2} \neq 0$, we have that for $\epsilon \rightarrow 0$

$$
\operatorname{det} \beta_{m+3}=O\left(\epsilon^{r}\right) \Leftrightarrow \operatorname{det} Z_{3} \neq 0 .
$$

(4) If det $D_{m, 0} \neq 0$, det $Z_{1} \neq 0$, det $Z_{2} \neq 0$ and $\operatorname{det} Z_{3} \neq 0$ we have that

$$
\operatorname{det} \beta_{m+4}=O(1), \quad \epsilon \rightarrow 0,
$$

generically. 
Proof. See appendix B.

The matrices $Z_{1}, Z_{2}$ and $Z_{3}$ can be expressed in terms of initial conditions as follows.

Proposition 2. The following expressions in terms of initial conditions hold:

$$
\begin{aligned}
Z_{1}= & m D_{m, 0}^{-1}-D_{m-1}-D_{m, 0}-\alpha_{22}-D_{m, 0}^{-1} C_{m, 0}\left(B_{m-1}+\alpha_{12}\right), \\
Z_{2}= & (m+1)\left(m D_{m, 0}^{-1}-D_{m, 0}^{-1} C_{m, 0}\left(B_{m-1}+\alpha_{12}\right)-D_{m-1}-D_{m, 0}-\alpha_{22}\right)^{-1} \\
& +D_{m, 0}^{-1} C_{m, 0} B_{m-1}-m D_{m, 0}^{-1}+D_{m-1}, \\
Z_{3}= & D_{m, 0}-(m+1) Z_{1}^{-1}+(m+2) Z_{2}^{-1} .
\end{aligned}
$$

Proof. Is a byproduct of the proof of theorem 2.

\section{Appendix A. Schur complements}

To show the genericness of the confinement phenomenon in the non- Abelian scenario it is very convenient to introduce Schur complements.

Definition 4. Given $M$ in the block form as in (13), the Schur complements with respect to $D$ (if $\operatorname{det} D \neq 0$ ), and to $A$ (if $\operatorname{det} A \neq 0$ ) are defined to be

$$
S_{D}(M):=A-B D^{-1} C, \quad S_{A}(M):=D-C A^{-1} B,
$$

respectively.

In terms of the Schur complements we have the following well-known expressions for the inverse matrices

$$
\begin{aligned}
& M^{-1}= \\
& \left\{\begin{array}{cc}
\left(\begin{array}{cc}
S_{D}(M)^{-1} & -S_{D}(M)^{-1} B D^{-1} \\
-D^{-1} C S_{D}(M)^{-1} & D^{-1}\left(\mathbb{I}_{N-r}+C S_{D}(M)^{-1} B D^{-1}\right)
\end{array}\right), & \text { for } \operatorname{det} D, \operatorname{det} S_{D}(M) \neq 0, \\
\left(\begin{array}{cc}
A^{-1}+A^{-1} B S_{A}(M)^{-1} C A^{-1} & -A^{-1} B S_{A}(M)^{-1} \\
-S_{A}(M)^{-1} C A^{-1} & S_{A}(M)^{-1}
\end{array}\right), & \text { for } \operatorname{det} A, \operatorname{det} S_{A}(M) \neq 0, \\
\left(\begin{array}{cc}
S_{D}(M)^{-1} & -S_{D}(M)^{-1} B D^{-1} \\
-D^{-1} C S_{D}(M)^{-1} & S_{A}(M)^{-1}
\end{array}\right), & \text { for } \operatorname{det} A, \operatorname{det} D, \operatorname{det} S_{D}(M),
\end{array}\right.
\end{aligned}
$$

and for the determinant of $M$

$$
\begin{aligned}
& \operatorname{det} M=\operatorname{det} A \quad \operatorname{det} S_{A}(M) \\
& =\operatorname{det} D \quad \operatorname{det} S_{D}(M) .
\end{aligned}
$$

Now, if $K=\left(\begin{array}{ll}A & B \\ C & D\end{array}\right)=\left(\begin{array}{cc}0 & 0 \\ C_{0} & D_{0}\end{array}\right)+\left(\begin{array}{ll}A_{1} & B_{1} \\ C_{1} & D_{1}\end{array}\right) \epsilon+O\left(\epsilon^{2}\right) \in \mathcal{A}_{\mathfrak{K}}$ then we can write the Schur complements in the form

$$
\begin{aligned}
& S_{D}(K)=A-B D^{-1} C=: S_{D}(K)_{1} \epsilon+S_{D}(K)_{2} \epsilon^{2}+O\left(\epsilon^{3}\right), \quad \epsilon \rightarrow 0, \\
& S_{A}(K)=D-C A^{-1} B=: S_{A}(K)_{0}+S_{A}(K)_{1} \epsilon+O\left(\epsilon^{2}\right), \quad \epsilon \rightarrow 0,
\end{aligned}
$$


where

$$
\begin{aligned}
S_{D}(K)_{1}= & A_{1}-B_{1} D_{0}^{-1} C_{0}, \\
S_{D}(K)_{2}= & A_{2}-B_{1} D_{0}^{-1} C_{1}-B_{2} D_{0}^{-1} C_{0}+B_{1} D_{0}^{-1} D_{1} D_{0}^{-1} C_{0}, \\
S_{D}(K)_{3}= & A_{3}-B_{1} D_{0}^{-1} C_{2}+\left(B_{1} D_{0}^{-1} D_{1} D_{0}^{-1}-B_{2} D_{0}^{-1}\right) C_{1} \\
& +B_{1}\left(D_{0}^{-1} D_{2} D_{0}^{-1}-D_{0}^{-1} D_{1} D_{0}^{-1} D_{1} D_{0}^{-1}\right) C_{0}+B_{2} D_{0}^{-1} D_{1} D_{0}^{-1} C_{0}-B_{3} D_{0}^{-1} C_{0}, \\
S_{D}(K)_{4}= & A_{4}-B_{1} D_{0}^{-1} C_{3}+B_{1} D_{0}^{-1} D_{1} D_{0}^{-1} C_{2}-B_{1} D_{0}^{-1}\left(D_{1} D_{0}^{-1} D_{1} D_{0}^{-1}-D_{2} D_{0}^{-1}\right) C_{1} \\
& -B_{1} D_{0}^{-1} D_{2} D_{0}^{-1} D_{1} D_{0}^{-1} C_{0}+B_{1} D_{0}^{-1} D_{1}\left(D_{0}^{-1} D_{1} D_{0}^{-1} D_{1} D_{0}^{-1}-D_{0}^{-1} D_{2} D_{0}^{-1}\right) C_{0} \\
& +B_{1} D_{0}^{-1} D_{3} D_{0}^{-1} C_{0}-B_{2} D_{0}^{-1} C_{2}+B_{2} D_{0}^{-1} D_{1} D_{0}^{-1} C_{1} \\
& -B_{2} D_{0}^{-1}\left(D_{1} D_{0}^{-1} D_{1} D_{0}^{-1}-D_{2} D_{0}^{-1}\right) C_{0}-B_{3} D_{0}^{-1}\left(C_{1}-D_{1} D_{0}^{-1} C_{0}\right)-B_{4} D_{0}^{-1} C_{0}, \\
S_{A}(K)_{0}= & D_{0}-C_{0} A_{1}^{-1} B_{1}, \\
S_{A}(K)_{1}= & D_{1}-C_{0} A_{1}^{-1} B_{2}-C_{1} A_{1}^{-1} B_{1}+C_{0} A_{1}^{-1} A_{2} A_{1}^{-1} B_{1} .
\end{aligned}
$$

For the determinant det $M$ we just take into account equations (23) and (24) to obtain $\operatorname{det} K=\epsilon^{r} \operatorname{det}\left(A_{1}-B_{1} D_{0}^{-1} C_{0}+O(\epsilon)\right) \operatorname{det}\left(D_{0}+O(\epsilon)\right)$

$$
=\operatorname{det}\left(A_{1}-B_{1} D_{0}^{-1} C_{0}\right) \operatorname{det}\left(D_{0}\right) \epsilon^{r}+O\left(\epsilon^{r+1}\right) .
$$

\section{Appendix B. Proof of theorem 2}

Lemma 2. (1) Assuming that $\operatorname{det} D_{m, 0} \neq 0$ the following asymptotic holds.

$$
\begin{aligned}
\operatorname{det} \beta_{m+1}= & \epsilon^{-r}\left|\begin{array}{lc}
m S_{D}\left(\beta_{m}\right)_{1}^{-1} & -m S_{D}\left(\beta_{m}\right)_{1}^{-1} B_{m, 1} D_{m, 0}^{-1}-B_{m-1}-\alpha_{12} \\
-m D_{m, 0}^{-1} C_{m, 0} S_{D}\left(\beta_{m}\right)_{1}^{-1} & m D_{m, 0}^{-1}+m D_{m, 0}^{-1} C_{m, 0} S_{D}\left(\beta_{m}\right)_{1}^{-1} B_{m, 1} D_{m, 0}^{-1} \\
-D_{m-1}-D_{m, 0}-\alpha_{22}
\end{array}\right| \\
& +O\left(\epsilon^{-r+1}\right)
\end{aligned}
$$

Proof. From equation (7) we know that

$$
\operatorname{det}\left(\begin{array}{cc}
A_{m, 1} & B_{m, 1} \\
C_{m, 0} & D_{m, 0}
\end{array}\right) \neq 0
$$

hence $S_{D}\left(\beta_{m}\right)_{1}$ is invertible. Then, from (22) and (24) we deduce

$$
\begin{aligned}
& \beta_{m}^{-1}=\left(\begin{array}{ll}
\left(\beta_{m}^{-1}\right)_{11,-1} & 0 \\
\left(\beta_{m}^{-1}\right)_{21,-1} & 0
\end{array}\right) \epsilon^{-1}+\left(\begin{array}{ll}
\left(\beta_{m}^{-1}\right)_{11,0} & \left(\beta_{m}^{-1}\right)_{12,0} \\
\left(\beta_{m}^{-1}\right)_{21,0} & \left(\beta_{m}^{-1}\right)_{22,0}
\end{array}\right) \\
& +\left(\begin{array}{ll}
\left(\beta_{m}^{-1}\right)_{11,1} & \left(\beta_{m}^{-1}\right)_{12,1} \\
\left(\beta_{m}^{-1}\right)_{21,1} & \left(\beta_{m}^{-1}\right)_{22,1}
\end{array}\right) \epsilon+O\left(\epsilon^{2}\right), \quad \epsilon \rightarrow 0,
\end{aligned}
$$

where the pole coefficients are

$$
\left(\beta_{m}^{-1}\right)_{11,-1}:=S_{D}\left(\beta_{m}\right)_{1}^{-1}, \quad\left(\beta_{m}^{-1}\right)_{21,-1}:=-D_{m, 0}^{-1} C_{m, 0} S_{D}\left(\beta_{m}\right)_{1}^{-1},
$$

while the regular part coefficients are

$$
\begin{aligned}
& \left(\beta_{m}^{-1}\right)_{11,0}:=-S_{D}\left(\beta_{m}\right)_{1}^{-1} S_{D}\left(\beta_{m}\right)_{2} S_{D}\left(\beta_{m}\right)_{1}^{-1}, \\
& \left(\beta_{m}^{-1}\right)_{12,0}:=-S_{D}\left(\beta_{m}\right)_{1}^{-1} B_{m, 1} D_{m, 0}^{-1}, \\
& \left(\beta_{m}^{-1}\right)_{21,0}:=D_{m, 0}^{-1}\left(C_{m, 0} S_{D}\left(\beta_{m}\right)_{1}^{-1} S_{D}\left(\beta_{m}\right)_{2} S_{D}\left(\beta_{m}\right)_{1}^{-1}-\left(C_{m, 1}-D_{m, 1} D_{m, 0}^{-1} C_{m, 0}\right) S_{D}\left(\beta_{m}\right)_{1}^{-1}\right), \\
& \left(\beta_{m}^{-1}\right)_{22,0}:=D_{m, 0}^{-1}\left(\mathbb{I}_{N-r}+C_{m, 0} S_{D}\left(\beta_{m}\right)_{1}^{-1} B_{m, 1} D_{m, 0}^{-1}\right),
\end{aligned}
$$




$$
\begin{aligned}
& \left(\beta_{m}^{-1}\right)_{11,1}:=S_{D}\left(\beta_{m}\right)_{1}^{-1} S_{D}\left(\beta_{m}\right)_{2} S_{D}\left(\beta_{m}\right)_{1}^{-1} S_{D}\left(\beta_{m}\right)_{2} S_{D}\left(\beta_{m}\right)_{1}^{-1}-S_{D}\left(\beta_{m}\right)_{1}^{-1} S_{D}\left(\beta_{m}\right)_{3} S_{D}\left(\beta_{m}\right)_{1}^{-1}, \\
& \left(\beta_{m}^{-1}\right)_{12,1}:=\left(S_{D}\left(\beta_{m}\right)_{1}^{-1} S_{D}\left(\beta_{m}\right)_{2} S_{D}\left(\beta_{m}\right)_{1}^{-1} B_{m, 1}-S_{D}\left(\beta_{m}\right)_{1}^{-1}\left(B_{m, 2}-B_{m, 1} D_{m, 0}^{-1} D_{m, 1}\right)\right) D_{m, 0}^{-1} \text {, } \\
& \left(\beta_{m}^{-1}\right)_{21,1}:=-D_{m, 0}^{-1}\left(C _ { m , 0 } \left[S_{D}\left(\beta_{m}\right)_{1}^{-1} S_{D}\left(\beta_{m}\right)_{2} S_{D}\left(\beta_{m}\right)_{1}^{-1} S_{D}\left(\beta_{m}\right)_{2} S_{D}\left(\beta_{m}\right)_{1}^{-1}\right.\right. \\
& \left.-S_{D}\left(\beta_{m}\right)_{1}^{-1} S_{D}\left(\beta_{m}\right)_{3} S_{D}\left(\beta_{m}\right)_{1}^{-1}\right] \\
& -\left(C_{m, 1}-D_{m, 1} D_{m, 0}^{-1} C_{m, 0}\right) S_{D}\left(\beta_{m}\right)_{1}^{-1} S_{D}\left(\beta_{m}\right)_{2} S_{D}\left(\beta_{m}\right)_{1}^{-1}+ \\
& \text { - } \left.\left(\left(D_{m, 1} D_{m, 0}^{-1} D_{m, 1}-D_{m, 2}\right) D_{m, 0}^{-1} C_{m, 0}+C_{m, 2}-D_{m, 1} D_{m, 0}^{-1} C_{m, 1}\right) S_{D}\left(\beta_{m}\right)_{1}^{-1}\right), \\
& \left(\beta_{m}^{-1}\right)_{22,1}:=D_{m, 0}^{-1}\left(-D_{m, 1}+\left(C_{m, 1}-D_{m, 1} D_{m, 0}^{-1} C_{m, 0}-C_{m, 0} S_{D}\left(\beta_{m}\right)_{1}^{-1} S_{D}\left(\beta_{m}\right)_{2}\right) S_{D}\left(\beta_{m}\right)_{1}^{-1} B_{m, 1}\right. \\
& \left.+C_{m, 0} S_{D}\left(\beta_{m}\right)_{1}^{-1}\left(B_{m, 2}-B_{m, 1} D_{m, 0}^{-1} D_{m, 1}\right)\right) D_{m, 0}^{-1}, \\
& \left(\beta_{m}^{-1}\right)_{11,2}:=S_{D}\left(\beta_{m}\right)_{1}^{-1} S_{D}\left(\beta_{m}\right)_{3} S_{D}\left(\beta_{m}\right)_{1}^{-1} S_{D}\left(\beta_{m}\right)_{2} S_{D}\left(\beta_{m}\right)_{1}^{-1} \\
& -S_{D}\left(\beta_{m}\right)_{1}^{-1} S_{D}\left(\beta_{m}\right)_{2} S_{D}\left(\beta_{m}\right)_{1}^{-1}\left(S_{D}\left(\beta_{m}\right)_{2} S_{D}\left(\beta_{m}\right)_{1}^{-1} S_{D}\left(\beta_{m}\right)_{2} S_{D}\left(\beta_{m}\right)_{1}^{-1}\right. \\
& \left.-S_{D}\left(\beta_{m}\right)_{3} S_{D}\left(\beta_{m}\right)_{1}^{-1}\right)-S_{D}\left(\beta_{m}\right)_{1}^{-1} S_{D}\left(\beta_{m}\right)_{4} S_{D}\left(\beta_{m}\right)_{1}^{-1}, \\
& \left(\beta_{m}^{-1}\right)_{12,2}:=S_{D}\left(\beta_{m}\right)_{1}^{-1} B_{m, 1} D_{m, 0}^{-1}\left(D_{m, 2} D_{m, 0}^{-1}-D_{m, 1} D_{m, 0}^{-1} D_{m, 1} D_{m, 0}^{-1}\right)+S_{D}\left(\beta_{m}\right)_{1}^{-1}\left(B_{m, 2}\right. \\
& \left.-S_{D}\left(\beta_{m}\right)_{2} S_{D}\left(\beta_{m}\right)_{1}^{-1} B_{m, 1}\right) D_{m, 0}^{-1} D_{m, 1} D_{m, 0}^{-1} \\
& -S_{D}\left(\beta_{m}\right)_{1}^{-1}\left(B_{m, 3}-S_{D}\left(\beta_{m}\right)_{2} S_{D}\left(\beta_{m}\right)_{1}^{-1} B_{m, 2}\right. \\
& \left.+S_{D}\left(\beta_{m}\right)_{2} S_{D}\left(\beta_{m}\right)_{1}^{-1} S_{D}\left(\beta_{m}\right)_{2} S_{D}\left(\beta_{m}\right)_{1}^{-1} B_{m, 1}-S_{D}\left(\beta_{m}\right)_{3} S_{D}\left(\beta_{m}\right)_{1}^{-1} B_{m, 1}\right) D_{m, 0}^{-1} .
\end{aligned}
$$

Finally, from equation (1) we deduce

$$
\begin{gathered}
\beta_{m+1}=\left(\begin{array}{cc}
m S_{D}\left(\beta_{m}\right)_{1}^{-1} & 0 \\
-m D_{m, 0}^{-1} C_{m, 0} S_{D}\left(\beta_{m}\right)_{1}^{-1} & 0
\end{array}\right) \epsilon^{-1}+\left(\begin{array}{ll}
A_{m+1,0} & B_{m+1,0} \\
C_{m+1,0} & D_{m+1,0}
\end{array}\right) \\
+\left(\begin{array}{ll}
A_{m+1,1} & B_{m+1,1} \\
C_{m+1,1} & D_{m+1,1}
\end{array}\right) \epsilon+O\left(\epsilon^{2}\right), \epsilon \rightarrow 0
\end{gathered}
$$

where

$$
\begin{aligned}
& A_{m+1,0}:=m\left(\beta_{m}^{-1}\right)_{11,0}-A_{m-1}-\alpha_{11}, \quad B_{m+1,0}:=m\left(\beta_{m}^{-1}\right)_{12,0}-B_{m-1}-\alpha_{12}, \\
& C_{m+1,0}:=m\left(\beta_{m}^{-1}\right)_{21,0}-C_{m-1}-C_{m, 0}-\alpha_{21}, \quad D_{m+1,0}:=m\left(\beta_{m}^{-1}\right)_{22,0}-D_{m-1}-D_{m, 0}-\alpha_{22}, \\
& A_{m+1,1}:=m\left(\beta_{m}^{-1}\right)_{11,1}-A_{m-1}-A_{m, 1}, \quad B_{m+1,1}:=m\left(\beta_{m}^{-1}\right)_{12,1}-B_{m-1}-B_{m, 1}, \\
& C_{m+1,1}:=m\left(\beta_{m}^{-1}\right)_{21,1}-C_{m, 1}, \quad D_{m+1,1}:=m\left(\beta_{m}^{-1}\right)_{22,1}-D_{m, 1}, \\
& A_{m+1,2}:=m\left(\beta_{m}^{-1}\right)_{11,2}-A_{m, 2}, \quad B_{m+1,2}:=m\left(\beta_{m}^{-1}\right)_{12,2}-B_{m, 2} .
\end{aligned}
$$

Observing that

$$
\operatorname{det} \beta_{m+1}=\left|\begin{array}{cc}
m S_{D}\left(\beta_{m}\right)_{1}^{-1} & B_{m+1,0} \\
-m D_{m, 0}^{-1} C_{m, 0} S_{D}\left(\beta_{m}\right)_{1}^{-1} & D_{m+1,0}
\end{array}\right| \epsilon^{-r}+O\left(\epsilon^{-r+1}\right), \quad \epsilon \rightarrow 0,
$$

the result follows.

Now observe that

$$
\begin{aligned}
Z_{1}:= & m D_{m, 0}^{-1}+m D_{m, 0}^{-1} C_{m, 0} S_{D}\left(\beta_{m}\right)_{1}^{-1} B_{m, 1} D_{m, 0}^{-1}-D_{m-1}-D_{m, 0}-\alpha_{22} \\
& -\left(-m D_{m, 0}^{-1} C_{m, 0} S_{D}\left(\beta_{m}\right)_{1}^{-1}\right)\left(m S_{D}\left(\beta_{m}\right)_{1}^{-1}\right)^{-1}\left(-m S_{D}\left(\beta_{m}\right)_{1}^{-1} B_{m, 1} D_{m, 0}^{-1}-B_{m-1}-\alpha_{12}\right) \\
= & m D_{m, 0}^{-1}-D_{m-1}-D_{m, 0}-\alpha_{22}-D_{m, 0}^{-1} C_{m, 0}\left(B_{m-1}+\alpha_{12}\right) .
\end{aligned}
$$


Using the determinant expansion in Schur complements of lemma 2, one observes that

$$
\begin{gathered}
\left|\begin{array}{cc}
m S_{D}\left(\beta_{m}\right)_{1}^{-1} & -m S_{D}\left(\beta_{m}\right)_{1}^{-1} B_{m, 1} D_{m, 0}^{-1}-B_{m-1}-\alpha_{12} \\
-m D_{m, 0}^{-1} C_{m, 0} S_{D}\left(\beta_{m}\right)_{1}^{-1} & m D_{m, 0}^{-1}+m D_{m, 0}^{-1} C_{m, 0} S_{D}\left(\beta_{m}\right)_{1}^{-1} B_{m, 1} D_{m, 0}^{-1}-D_{m-1}-D_{m, 0}-\alpha_{22}
\end{array}\right| \\
=\operatorname{det}\left(m S_{D}\left(\beta_{m}\right)_{1}^{-1}\right) \operatorname{det} Z_{1} .
\end{gathered}
$$

and the first point of the theorem is proved.

Let us now go one step further in the discrete matrix chain and move to position $m+2$.

Lemma 3. Whenever $\operatorname{det} D_{m, 0} \neq 0$ and $\operatorname{det} Z_{1} \neq 0$ the following asymptotic hold.

$$
\begin{aligned}
\operatorname{det} \beta_{m+2}= & \epsilon^{-r}\left|\begin{array}{ll}
-m S_{D}\left(\beta_{m}\right)_{1}^{-1} & m S_{D}\left(\beta_{m}\right)_{1}^{-1} B_{m, 1} D_{m, 0}^{-1}+B_{m-1} \\
m D_{m, 0}^{-1} C_{m, 0} S_{D}\left(\beta_{m}\right)_{1}^{-1} & (m+1) Z_{1}^{-1}-m D_{m, 0}^{-1} \\
& -m D_{m, 0}^{-1} C_{m, 0} S_{D}\left(\beta_{m}\right)_{1}^{-1} B_{m, 1} D_{m, 0}^{-1}+D_{m-1}
\end{array}\right| \\
& +O\left(\epsilon^{-r+1}\right)
\end{aligned}
$$

for $\epsilon \rightarrow 0$.

Proof. As det $\beta_{m+1}=O\left(\epsilon^{-r}\right), \epsilon \rightarrow 0$, and consequently point (2) of proposition 1 tells us that $\beta_{m+1}^{-1} \in \mathcal{A}_{\mathfrak{K}}$. Therefore, the following asymptotic expansion for the inverse matrix holds

$$
\begin{gathered}
\beta_{m+1}^{-1}=\left(\begin{array}{cc}
0 & 0 \\
\left(\beta_{m+1}^{-1}\right)_{21,0} & \left(\beta_{m+1}^{-1}\right)_{22,0}
\end{array}\right)+\left(\begin{array}{ll}
\left(\beta_{m+1}^{-1}\right)_{11,1} & \left(\beta_{m+1}^{-1}\right)_{12,1} \\
\left(\beta_{m+1}^{-1}\right)_{21,1} & \left(\beta_{m+1}^{-1}\right)_{22,1}
\end{array}\right) \epsilon \\
+\left(\begin{array}{cc}
\left(\beta_{m+1}^{-1}\right)_{11,2} & \left(\beta_{m+1}^{-1}\right)_{12,2} \\
\left(\beta_{m+1}^{-1}\right)_{21,2} & \left(\beta_{m+1}^{-1}\right)_{22,2}
\end{array}\right) \epsilon^{2}+O\left(\epsilon^{3}\right),
\end{gathered}
$$

for $\epsilon \rightarrow 0$. Here the blocks $\left(\beta_{m+1}^{-1}\right)_{a b, j}$ are to be found from the asymptotic expansion (26). We conclude

$$
\begin{aligned}
&\left(\beta_{m+1}^{-1}\right)_{21,0}= Z_{1}^{-1} D_{m, 0}^{-1} C_{m, 0}, \quad\left(\beta_{m+1}^{-1}\right)_{22,0}=Z_{1}^{-1} \\
&\left(\beta_{m+1}^{-1}\right)_{11,1}= \frac{1}{m} S_{D}\left(\beta_{m}\right)_{1}-\frac{1}{m} S_{D}\left(\beta_{m}\right)_{1} B_{m+1,0} Z_{1}^{-1} D_{m, 0}^{-1} C_{m, 0} \\
&\left(\beta_{m+1}^{-1}\right)_{12,1}=-\frac{1}{m} S_{D}\left(\beta_{m}\right)_{1} B_{m+1,0} Z_{1}^{-1} \\
&\left(\beta_{m+1}^{-1}\right)_{21,1}=-Z_{1}^{-1} D_{m, 0}^{-1} C_{m, 0}-\frac{1}{m} Z_{1}^{-1}\left(C_{m+1,0}+D_{m, 0}^{-1} C_{m, 0} A_{m+1,0}\right) S_{D}\left(\beta_{m}\right)_{1} \\
& \times\left(\mathbb{I}_{r}-B_{m+1,0} Z_{1}^{-1} D_{m, 0}^{-1} C_{m, 0}\right) \\
&\left(\beta_{m+1}^{-1}\right)_{22,1}=-Z_{1}^{-1}+\frac{1}{m} Z_{1}^{-1}\left(C_{m+1,0}+D_{m, 0}^{-1} C_{m, 0} A_{m+1,0}\right) S_{D}\left(\beta_{m}\right)_{1} B_{m+1,0} Z_{1}^{-1} \\
&\left(\beta_{m+1}^{-1}\right)_{11,2}=-\frac{1}{m^{2}} S_{D}\left(\beta_{m}\right)_{1} A_{m+1,0} S_{D}\left(\beta_{m}\right)_{1}+\frac{1}{m^{2}} S_{D}\left(\beta_{m}\right)_{1} A_{m+1,0} S_{D}\left(\beta_{m}\right)_{1} B_{m+1,0} Z_{1}^{-1} D_{m, 0}^{-1} C_{m, 0} \\
&+\frac{1}{m^{2}} S_{D}\left(\beta_{m}\right)_{1} B_{m+1,0} Z_{1}^{-1}\left(C_{m+1,0}+D_{m, 0}^{-1} C_{m, 0} A_{m+1,0}\right) S_{D}\left(\beta_{m}\right)_{1}\left(\mathbb{I}_{r}-B_{m+1,0} Z_{1}^{-1} D_{m, 0}^{-1} C_{m, 0}\right) \\
&\left(\beta_{m+1}^{-1}\right)_{12,2}=- \frac{1}{m^{2}} S_{D}\left(\beta_{m}\right)_{1} B_{m+1,0} Z_{1}^{-1}\left(C_{m+1,0}+D_{m, 0}^{-1} C_{m, 0} A_{m+1,0}\right) S_{D}\left(\beta_{m}\right)_{1} B_{m+1,0} Z_{1}^{-1} \\
&+\frac{1}{m^{2}} S_{D}\left(\beta_{m}\right)_{1} A_{m+1,0} S_{D}\left(\beta_{m}\right)_{1} B_{m+1,0} Z_{1}^{-1}
\end{aligned}
$$


If we substitute equations (27)-(31) into equation (1), we have that for $\epsilon \rightarrow 0$

$$
\begin{aligned}
& \beta_{m+2}=\left(\begin{array}{cc}
-m S_{D}\left(\beta_{m}\right)_{1}^{-1} & 0 \\
m D_{m, 0}^{-1} C_{m, 0} S_{D}\left(\beta_{m}\right)_{1}^{-1} & 0
\end{array}\right) \epsilon^{-1}+\left(\begin{array}{ll}
A_{m+2,0} & B_{m+2,0} \\
C_{m+2,0} & D_{m+2,0}
\end{array}\right) \\
& +\left(\begin{array}{ll}
A_{m+2,1} & B_{m+2,1} \\
C_{m+2,1} & D_{m+2,1}
\end{array}\right) \epsilon+O\left(\epsilon^{2}\right),
\end{aligned}
$$

where

$$
\begin{aligned}
A_{m+2,0}:=-A_{m+1,0}-\alpha_{11}, \quad B_{m+2,0}:=-B_{m+1,0}-\alpha_{12}, \\
C_{m+2,0}:=(m+1)\left(\beta_{m+1}^{-1}\right)_{21,0}-C_{m+1,0}-C_{m, 0}-\alpha_{21}, \\
D_{m+2,0}:=(m+1)\left(\beta_{m+1}^{-1}\right)_{22,0}-D_{m+1,0}-D_{m, 0}-\alpha_{22}, \\
A_{m+2,1}:=(m+1)\left(\beta_{m+1}^{-1}\right)_{11,1}-A_{m+1,1}-A_{m, 1}, \\
B_{m+2,1}:=(m+1)\left(\beta_{m+1}^{-1}\right)_{12,1}-B_{m+1,1}-B_{m, 1}, \\
C_{m+2,1}:=(m+1)\left(\beta_{m+1}^{-1}\right)_{21,1}-C_{m+1,1}-C_{m, 1}, \\
D_{m+2,1}:=(m+1)\left(\beta_{m+1}^{-1}\right)_{22,1}-D_{m+1,1}-D_{m, 1}, \\
A_{m+2,2}:=(m+1)\left(\beta_{m+1}^{-1}\right)_{11,2}-A_{m+1,2}-A_{m, 2}, \\
B_{m+2,2}:=(m+1)\left(\beta_{m+1}^{-1}\right)_{12,2}-B_{m+1,2}-B_{m, 2} .
\end{aligned}
$$

Now, observing that

$$
\operatorname{det} \beta_{m+2}=\left|\begin{array}{cc}
-m S_{D}\left(\beta_{m}\right)_{1}^{-1} & B_{m+2,0} \\
m D_{m, 0}^{-1} C_{m, 0} S_{D}\left(\beta_{m}\right)_{1}^{-1} & D_{m+2,0}
\end{array}\right| \epsilon^{-r}+O\left(\epsilon^{-r+1}\right), \quad \epsilon \rightarrow 0,
$$

the result follows.

Note that

$$
\begin{aligned}
Z_{2}:= & (m+1)\left(m D_{m, 0}^{-1}-D_{m, 0}^{-1} C_{m, 0}\left(B_{m-1}+\alpha_{12}\right)-D_{m-1}-D_{m, 0}-\alpha_{22}\right)^{-1} \\
& +D_{m, 0}^{-1} C_{m, 0} B_{m-1}-m D_{m, 0}^{-1}+D_{m-1} .
\end{aligned}
$$

We expand the determinant according to Schur complements, obtaining

$$
\begin{gathered}
\left|\begin{array}{cc}
-m S_{D}\left(\beta_{m}\right)_{1}^{-1} & m S_{D}\left(\beta_{m}\right)_{1}^{-1} B_{m, 1} D_{m, 0}^{-1}+B_{m-1} \\
m D_{m, 0}^{-1} C_{m, 0} S_{D}\left(\beta_{m}\right)_{1}^{-1} & (m+1) Z_{1}^{-1}-m D_{m, 0}^{-1}-m D_{m, 0}^{-1} C_{m, 0} S_{D}\left(\beta_{m}\right)_{1}^{-1} B_{m, 1} D_{m, 0}^{-1}+D_{m-1}
\end{array}\right| \\
=\operatorname{det}\left(\begin{array}{c}
\left.-m S_{D}\left(\beta_{m}\right)_{1}^{-1}\right) \operatorname{det} Z_{2}
\end{array}\right.
\end{gathered}
$$

from which the second point of the theorem follows immediately.

Lemma 4. Assuming that $\operatorname{det} D_{m, 0} \neq 0$, det $Z_{1} \neq 0$ and $\operatorname{det} Z_{2} \neq 0$ the following asymptotic expansion for $\epsilon \rightarrow 0$ holds

$$
\begin{aligned}
& \operatorname{det} \beta_{m+3}=\epsilon^{r}\left|\begin{array}{lc}
(m+2)\left(\beta_{m+2}^{-1}\right)_{11,1} & (m+2)\left(\beta_{m+2}^{-1}\right)_{12,1} \\
-(m+1)\left(\beta_{m+1}^{-1}\right)_{11,1}+A_{m, 1} & -(m+1)\left(\beta_{m+1}^{-1}\right)_{12,1}+B_{m, 1} \\
(m+2)\left(\beta_{m+2}^{-1}\right)_{21,0} & (m+2)\left(\beta_{m+2}^{-1}\right)_{22,0} \\
-(m+1)\left(\beta_{m+1}^{-1}\right)_{21,0}+C_{m, 0} & -(m+1)\left(\beta_{m+1}^{-1}\right)_{22,0}+D_{m, 0}
\end{array}\right| \\
& +O\left(\epsilon^{r+1}\right) \text {, }
\end{aligned}
$$

where

$$
\begin{aligned}
& \left(\beta_{m+2}^{-1}\right)_{21,0}:=Z_{2}^{-1} D_{m, 0}^{-1} C_{m, 0}, \quad\left(\beta_{m+2}^{-1}\right)_{22,0}:=Z_{2}^{-1}, \\
& \left(\beta_{m+2}^{-1}\right)_{11,1}:=-\frac{1}{m} S_{D}\left(\beta_{m}\right)_{1}\left(\mathbb{I}_{r}-B_{m+2,0} Z_{2}^{-1} D_{m, 0}^{-1} C_{m, 0}\right), \quad\left(\beta_{m+2}^{-1}\right)_{12,1}:=\frac{1}{m} S_{D}\left(\beta_{m}\right)_{1} B_{m+2,0} Z_{2}^{-1},
\end{aligned}
$$




$$
\begin{aligned}
\left(\beta_{m+2}^{-1}\right)_{21,1}:= & -Z_{2}^{-1} D_{m, 0}^{-1} C_{m, 0}+\frac{1}{m} Z_{2}^{-1}\left(C_{m+2,0}+D_{m, 0}^{-1} C_{m, 0} A_{m+2,0}\right) S_{D}\left(\beta_{m}\right)_{1} \\
& \times\left(\mathbb{I}_{r}-B_{m+2,0} Z_{2}^{-1} D_{m, 0}^{-1} C_{m, 0}\right) \\
\left(\beta_{m+2}^{-1}\right)_{22,1}:= & -Z_{2}^{-1}-\frac{1}{m} Z_{2}^{-1}\left(C_{m+2,0}+D_{m, 0}^{-1} C_{m, 0} A_{m+2,0}\right) S_{D}\left(\beta_{m}\right)_{1} B_{m+2,0} Z_{2}^{-1}, \\
\left(\beta_{m+2}^{-1}\right)_{11,2}:= & \frac{1}{m^{2}} S_{D}\left(\beta_{m}\right)_{1} B_{m+2,0} Z_{2}^{-1}\left(C_{m+2,0}+D_{m, 0}^{-1} C_{m, 0} A_{m+2,0}\right) S_{D}\left(\beta_{m}\right)_{1} \\
& \times\left(\mathbb{I}_{r}-B_{m+2,0} Z_{2}^{-1} D_{m, 0}^{-1} C_{m, 0}\right) \\
& -\frac{1}{m^{2}} S_{D}\left(\beta_{m}\right)_{1} A_{m+2,0} S_{D}\left(\beta_{m}\right)_{1}\left(\mathbb{I}_{r}-B_{m+2,0} Z_{2}^{-1} D_{m, 0}^{-1} C_{m, 0}\right), \\
\left(\beta_{m+2}^{-1}\right)_{12,2}:= & \frac{1}{m^{2}} S_{D}\left(\beta_{m}\right)_{1} A_{m+2,0} S_{D}\left(\beta_{m}\right)_{1} B_{m+2,0} Z_{2}^{-1} \\
& -\frac{1}{m^{2}} S_{D}\left(\beta_{m}\right)_{1} B_{m+2,0} Z_{2}^{-1}\left(C_{m+2,0}+D_{m, 0}^{-1} C_{m, 0} A_{m+2,0}\right) S_{D}\left(\beta_{m}\right)_{1} B_{m+2,0} Z_{2}^{-1} .
\end{aligned}
$$

Proof. From equation (33) we obtain that $\beta_{m+2} \in \mathbb{L}$. Therefore, since $\operatorname{det} Z_{2} \neq 0$, we have

$$
\begin{gathered}
\beta_{m+2}^{-1}=\left(\begin{array}{cc}
0 & 0 \\
\left(\beta_{m+2}^{-1}\right)_{21,0} & \left(\beta_{m+2}^{-1}\right)_{22,0}
\end{array}\right)+\left(\begin{array}{ll}
\left(\beta_{m+2}^{-1}\right)_{11,1} & \left(\beta_{m+2}^{-1}\right)_{12,1} \\
\left(\beta_{m+2}^{-1}\right)_{21,1} & \left(\beta_{m+2}^{-1}\right)_{22,1}
\end{array}\right) \epsilon \\
+\left(\begin{array}{cc}
\left(\beta_{m+2}^{-1}\right)_{11,2} & \left(\beta_{m+2}^{-1}\right)_{12,2} \\
\left(\beta_{m+2}^{-1}\right)_{21,2} & \left(\beta_{m+2}^{-1}\right)_{22,2}
\end{array}\right) \epsilon^{2}+O\left(\epsilon^{3}\right),
\end{gathered}
$$

where the blocks $\left(\beta_{m+2}^{-1}\right)_{a b, j}$ are determined by the asymptotic expansion (33). If we substitute (26), (33) and (34) into the matrix equation (1), we have that

$$
\beta_{m+3}=\left(\begin{array}{cc}
0 & 0 \\
C_{m+3,0} & D_{m+3,0}
\end{array}\right)+\left(\begin{array}{cc}
A_{m+3,1} & B_{m+3,1} \\
C_{m+3,1} & D_{m+3,1}
\end{array}\right) \epsilon+\left(\begin{array}{cc}
A_{m+3,2} & B_{m+3,2} \\
C_{m+3,2} & D_{m+3,2}
\end{array}\right) \epsilon^{2}+O\left(\epsilon^{3}\right),
$$

where

$$
\begin{gathered}
C_{m+3,0}:=(m+2)\left(\beta_{m+2}^{-1}\right)_{21,0}-(m+1)\left(\beta_{m+1}^{-1}\right)_{21,0}+C_{m, 0}, \\
D_{m+3,0}:=(m+2)\left(\beta_{m+2}^{-1}\right)_{22,0}-(m+1)\left(\beta_{m+1}^{-1}\right)_{22,0}+D_{m, 0}, \\
A_{m+3,1}:=(m+2)\left(\beta_{m+2}^{-1}\right)_{11,1}-(m+1)\left(\beta_{m+1}^{-1}\right)_{11,1}+A_{m, 1}, \\
B_{m+3,1}:=(m+2)\left(\beta_{m+2}^{-1}\right)_{12,1}-(m+1)\left(\beta_{m+1}^{-1}\right)_{12,1}+B_{m, 1}, \\
C_{m+3,1}:=(m+2)\left(\beta_{m+2}^{-1}\right)_{21,1}-(m+1)\left(\beta_{m+1}^{-1}\right)_{21,1}+C_{m, 1}, \\
D_{m+3,1}:=(m+2)\left(\beta_{m+2}^{-1}\right)_{22,1}-(m+1)\left(\beta_{m+1}^{-1}\right)_{22,1}+D_{m, 1}, \\
A_{m+3,2}:=(m+2)\left(\beta_{m+2}^{-1}\right)_{11,2}-(m+1)\left(\beta_{m+1}^{-1}\right)_{11,2}+A_{m, 2}, \\
B_{m+3,2}:=(m+2)\left(\beta_{m+2}^{-1}\right)_{12,2}-(m+1)\left(\beta_{m+1}^{-1}\right)_{12,2}+B_{m, 2} .
\end{gathered}
$$

Then, if we use again proposition 1 , we deduce

$$
\operatorname{det} \beta_{m+3}=\epsilon^{r}\left|\begin{array}{ll}
A_{m+3,1} & B_{m+3,1} \\
C_{m+3,0} & D_{m+3,0}
\end{array}\right|+O\left(\epsilon^{r+1}\right), \quad \epsilon \rightarrow 0,
$$

and the result follows.

Note that

$$
Z_{3}=D_{m, 0}-(m+1) Z_{1}^{-1}+(m+2) Z_{2}^{-1} .
$$

Note the similarity with equation (17). 
Taking into account that

$$
C_{m+3,0}=Z_{3} D_{m, 0}^{-1} C_{m, 0}, \quad D_{m+3,0}=Z_{3},
$$

we express the determinant in equation (35) as follows:

$$
\left|\begin{array}{ll}
A_{m+3,1} & B_{m+3,1} \\
C_{m+3,0} & D_{m+3,0}
\end{array}\right|=\operatorname{det} Z_{3} \operatorname{det}\left(A_{m+3,1}-B_{m+3,1} D_{m, 0}^{-1} C_{m, 0}\right),
$$

where

$$
A_{m+3,1}-B_{m+3,1} D_{m, 0}^{-1} C_{m, 0}=-\frac{(m+3)}{m} S_{D}\left(\beta_{m}\right)_{1} .
$$

This implies that the determinant in equation (35) vanishes if and only if

$$
\operatorname{det} Z_{3}=0 \text {. }
$$

Finally, under the previous hypotheses, equations (9)-(11) hold. As a by product of the proof of theorem 1, we obtain that

$$
\beta_{m+4}=\beta_{m+3}^{-1} A-\beta_{m+3}-\alpha
$$

where $\beta_{m+3}, A \in \mathcal{A}_{\mathfrak{K}}$ and $\left(\beta_{m+3}\right)^{-1} \in \epsilon^{-1} \mathcal{A}_{\mathfrak{L}}$. According to proposition $1(6), \beta_{m+3}^{-1} A \in \mathcal{A}$, so that we can write

$$
\beta_{m+4}=O(1), \quad \epsilon \rightarrow 0 .
$$

We can write the matrix dynamical system (1) as

$$
\beta_{n-1}=n \beta_{n}^{-1}-\beta_{n+1}-\beta_{n}-\alpha,
$$

which can be seen as the application of a time reversal symmetry. From $\beta_{m+4} \in \mathcal{A}$ and $\beta_{m+3} \in \mathcal{A}_{\mathfrak{K}}$, understood now as initial conditions, we obtain the quantities $\beta_{m+2}, \beta_{m+1}, \beta_{m}$ and $\beta_{m-1}$. Observe that our initial assumption was precisely that $\beta_{m-1} \in \mathcal{A}$ and $\beta_{m} \in \mathcal{A}_{\mathfrak{K}}$, see (7). Hence, the whole forward process, and its conclusions about the asymptotic behaviours, can be reversed backwards. Consequently, since the assumption that $\operatorname{det} \beta_{m+4,0}=0$ reduces the number of free parameters from $N^{2}$ to $N^{2}-1$, we conclude that $\beta_{m-1}$ involves at most $N^{2}-1$ free parameters (if no further constraint is requested). This is in contradiction to our departing hypothesis that $\beta_{m-1}$ has $N^{2}$ free parameters. Therefore $\operatorname{det} \beta_{m+4}=O(1)$ as $\epsilon \rightarrow 0$ generically.

\section{Acknowledgments}

PT has been supported by Spanish 'Ministerio de Ciencia e Innovación' grant FIS2011-00260. GC-C benefitted from the financial support of a 'Acción Especial' Ref. AE1/13-18837 of the Universidad Complutense de Madrid. MM acknowledges economical support from the Spanish 'Ministerio de Economía y Competitividad' research project MTM2012-36732-C0301, Ortogonalidad y aproximacion; Teoria y Aplicaciones.

\section{References}

[1] Ablowitz M J, Halburd R and Herbst B 2000 On the extension of the Painlevé property to difference equations Nonlinearity 13 889-905

[2] Adler M, van Moerbeke P and Vanhaecke P 2008 Singularity confinement for a class of $m$-th order difference equations of combinatorics Phil. Trans. R. Soc. Lond. A 366 877-922

[3] Arinkin D and Borodin A 2006 Moduli spaces of d-connections, difference Painlevé equations Duke Math. J. $134515-56$

[4] Bellon M P and Viallet C-M 1999 Algebraic entropy Commun. Math. Phys. 204 425-37 
[5] Bobenko A I and Suris Y 2008 Discrete differential geometry. Integrable structure Graduate Studies in Mathematics vol 98 (Providence, RI: American Mathematical Society) xxiv+404pp

[6] Bobenko A I and Suris Y 2002 Integrable systems on quad-graphs Int. Math. Res. Not. 11 573-611

[7] Cassatella G A and Mañas M 2012 Riemann-Hilbert problems, matrix orthogonal polynomals, discrete matrix equations with singularity confinement Stud. Appl. Math. 128 252-74

[8] Conte R (ed) 1999 The Painlevé Property. One Century Later (New York: Springer)

[9] Dynnikov I and Novikov S P 2003 Geometry of the triangle equation on two-manifolds Moscow Math. J. 3 419-38

[10] Fokas A S, Its A R and Kitaev A V 1991 Discrete Painlevé equations, their appearance in quantum gravity Commun. Math. Phys. 142 313-44

[11] Freud G 1976 On the coefficients in the recursion formulae of orthogonal polynomials Proc. R. Irish Acad. A $761-6$

[12] Gelfand I, Gelfand S, Retakh V and Wilson R 2005 Quasideterminants Adv. Math. 193 56-141

[13] Grammaticos B, Ramani A and Papageorgiou V 1991 Do integrable mappings have the Painlevé property? Phys. Rev. Lett. 67 1825-8

[14] Grünbaum F A, de la Iglesia M D and Martinez-Finkelshtein A 2011 Properties of matrix orthogonal polynomials via their Riemann-Hilbert characterization SIGMA 7098

[15] Hietarinta J and Viallet C 1998 Singularity confinement, chaos in discrete systems Phys. Rev. Lett. 81 325-8

[16] 't Hooft G 1996 Quantization of point particles in (2+1)-dimensional gravity and spacetime discreteness Class. Quantum Grav. 13 1023-39

[17] Lafortune S, Ramani A, Grammaticos B, Ohta Y and Tamizhmani K M 2001 Blending two discrete integrability criteria: singularity confinement and algebraic entropy Bäcklund and Darboux transformations. The geometry of solitons (Halifax, NS, 1999) (CRM Proceedings and Lecture Notes vol 29 (Providence, RI: American Mathematical Society) pp 299-311

[18] Moser J and Veselov A P 1991 Discrete versions of some classical integrable systems, factorization of matrix polynomials Commun. Math. Phys. $139217-43$

[19] Newman M E J 2010 Networks (Oxford: Oxford University Press)

[20] Novikov S P and Shvarts A S 1999 Discrete Lagrangian systems on graphs. Symplecto-topological properties Usp. Mat. Nauk $\mathbf{5 4}$ 257-58 (in Russian)

Novikov S P and Shvarts A S 1999 Math. Surv. 54 258-59 (Engl. transl.)

[21] Painlevé P 1973 Leçons sur la théorie analytique des équations différentielles (Leçons de Stockholm, delivered in 1895) Hermann, Paris (1897). Reprinted in Euvres de Paul Painlevé, vol I, Éditions du CNRS, Paris

[22] Ramani A, Grammaticos B, Tamizhmani T and Tamizhmani K M 2003 The road to the discrete analogue of the Painlevé property: Nevanlinna meets singularity confinement Comput. Math. Appl. 45 1001-12

[23] Suris Yu B 2003 The Problem of Integrable Discretization: Hamiltonian Approach (Progress in Mathematics vol 219) (Basel: Birkhäuser)

[24] Tempesta P 2013 Integrable maps from Galois differential algebras, Borel transforms, number sequences J. Diff. Eqns 255 2981-95

[25] Tsuda T 2009 Universal character and q-difference Painlevé equations Math. Ann. 345 395-415 